\title{
Jendrowiak, Hans-Werner
}

\section{Zur Dialektik von Soft Skills und fachlicher Kompetenz}

Hansel, Toni [Hrsg.]: Soft Skills. Alternative zur Fachlichkeit oder weiche Performance? Freiburg, Br. : Centaurus 2010, S. 21-43. - (Schulpädagogik; 10)

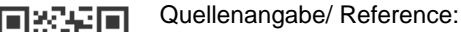

Jendrowiak, Hans-Werner: Zur Dialektik von Soft Skills und fachlicher Kompetenz - In: Hansel, Toni [Hrsg.]: Soft Skills. Alternative zur Fachlichkeit oder weiche Performance? Freiburg, Br. : Centaurus 2010, S. 21-43 - URN: urn:nbn:de:0111-opus-52961 - DOI: 10.25656/01:5296

https://nbn-resolving.org/urn:nbn:de:0111-opus-52961

https://doi.org/10.25656/01:5296

in Kooperation mit / in cooperation with:

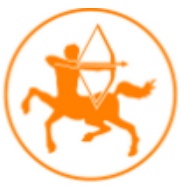

\section{CENTAURUS}

\section{Verlag \& Media KG}

\section{Nutzungsbedingungen}

Gewährt wird ein nicht exklusives, nicht übertragbares, persönliches und beschränktes Recht auf Nutzung dieses Dokuments. Dieses Dokument ist ausschließlich für den persönlichen, nicht-kommerziellen Gebrauch bestimmt. Die Nutzung stellt keine Übertragung des Eigentumsrechts an diesem Dokument dar und gilt vorbehaltlich der folgenden Einschränkungen: Auf sämtlichen Kopien dieses Dokuments müssen alle Urheberrechtshinweise und sonstigen Hinweise auf gesetzlichen Schutz beibehalten werden. Sie dürfen dieses Dokument nicht in irgendeiner Weise abändern, noch dürfen Sie dieses Dokument für öffentliche oder kommerzielle Zwecke vervielfältigen, öffentlich ausstellen, aufführen, vertreiben oder anderweitig nutzen.

Mit der Verwendung dieses Dokuments erkennen Sie die Nutzungsbedingungen an.

\section{Kontakt / Contact:}

\section{peDOCS}

DIPF | Leibniz-Institut für Bildungsforschung und Bildungsinformation Informationszentrum (IZ) Bildung

E-Mail: pedocs@dipf.de

Internet: www.pedocs.de

\section{Terms of use}

We grant a non-exclusive, non-transferable, individual and limited right to using this document.

This document is solely intended for your personal, non-commercial use. Use of this document does not include any transfer of property rights and it is conditional to the following limitations: All of the copies of this documents must retain all copyright information and other information regarding legal protection. You are not allowed to alter this document in any way, to copy it for public or commercial purposes, to exhibit the document in public, to perform, distribute or otherwise use the document in public.

By using this particular document, you accept the above-stated conditions of use. 


\section{Toni Hansel (Hg.)}

\section{Soft Skills}

\section{Alternative zur Fachlichkeit oder weiche Performance?}

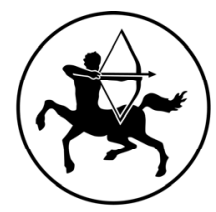

\section{CENTAURUS}


Der Herausgeber, Prof. Dr. Toni Hansel, ist Professor für Schulpädagogik an der Universität Rostock und Direktor des Instituts für Schulpädagogik.

Bibliografische Informationen der Deutschen Nationalbibliothek Die Deutsche Nationalbibliothek verzeichnet diese Publikation in der Deutschen Nationalbibliografie; detaillierte bibliografische Daten sind im Internet über http://dnb.d-nb.de abrufbar.

Gedruckt auf säurefreiem und chlorfrei gebleichtem Papier.

ISBN 978-3-86226-118-5

\section{ISSN 1616-7414}

Alle Rechte, insbesondere das Recht der Vervielfältigung und Verbreitung sowie der Übersetzung, vorbehalten. Kein Teil des Werkes darf in irgendeiner Form (durch Fotokopie, Mikrofilm oder ein anderes Verfahren) obne schriftliche Genehmigung des Verlages reproduziert oder unter Verwendung elektronischer Systeme verarbeitet, vervielfältigt oder verbreitet werden.

(C) CENTAURUS Verlag \& Media KG, Freiburg 2010

www.centaurus-verlag,. de

Umschlaggestaltung: Antje Walter, Titisee-Neustadt

Satz: Vorlage des Herausgebers 


\section{Zum Inhalt}

01

Vorwort

02

Stefan Göbel

Prorektor für Studium und Lehre

der Universität Rostock

03

Toni Hansel

Soft Skills -

Alternative zur Fachlichkeit oder weiche Performance?

\section{Geisteswissenschaftliche Grundlagen menschlicher Umgangsqualität}

04

Hans-Werner Jendrowiak

Zur Dialektik von Soft Skills und fachlicher Kompetenz

05

Wolfgang Sucharowski

Wissenskommunikation - erhöhen Skills die Kommunizierbarkeit von Wissen?

06

Gerhard Engel

Regeln, Ordnungen und Freiheit

Zur pädagogischen Relevanz von Thomas Hobbes' Sozialphilosophie 


\section{Vom Wissen zum Handeln in der Schule}

07

Alexander Steinhagen

„Kreativität als grundlegendes Soft-Skill im schulischen Bildungsprozess - zur Unabdingbarkeit dieser Leistungsdisposition für handlungskompetente Schüler“

08

Roland Straube

Soft Skills in der Lehrerbildung - Probleme und Folgen

09

Ulrika Gehrke

121

Soft-Skills in der Ausbildung der Gesundheitsfachberufe Kontinuität und Wandel

\section{Soft Skills und betriebliches Qualifika- tionsprofil: Vom Umgang mit Menschen}

Zur Bedeutung von Soft Skills für Beschäftigte in der Hotellerie und Gastronomie

Die Bedeutung von Schlüsselkompetenzen im der Berufspraxis am Beispiel Deutsche Bahn

Ausblick 


\section{Hans-Werner Jendrowiak}

\section{Zur Dialektik von Soft Skills und fachlicher Kompetenz}

\section{Die Problemstellung und die Bedeutsamkeit des Themas}

Es ist ein permanenter Prozess, der sich immer wieder mit der Frage nach Effektivität und Wirksamkeit von Bildung (Qualifikationen, Kompetenzen, Fähigkeiten und Fertigkeiten)beschäftigt. In das Zentrum betrieblicher Überlegungen rücken z.B. Aufgaben der Personalentwicklung und die Kosten des Personals. Das Bildungscontrolling, in seiner Funktion zur Überwachung und Evaluation von Bildungskosten stellt eine neue betriebswirtschaftliche Herausforderung dar. Die Diskussion wird auch über die Effektivität des Humankapitals geführt.

Es sind also nicht nur Fragen nach den Kosten, die Bildung verursachen, sondern auch Fragen nach dem Verhältnis von Bildungsaufwand und Bildungsnutzen, dem ökonomischen Prinzip. Dieses Prinzip, dem das Menschenbild des homo ökonomicus zu Grunde liegt, finden wir aber nicht nur in den originären ökonomischen Handlungsfeldern von Unternehmungen und Betrieben vor, den sogenannten profitorientierten Organisationen, sondern auch in der sogenannten Non-Profit-Organisation wie dem öffentlichen Bildungssystem.

Wir können drei Diskussionswege verfolgen:

- Erstens: Wenn die Mittel für Bildungs- und Personalentwicklungsmaßnahmen knapp werden, so ist die Pädagogik (Bildungswissenschaft, Erziehungswissenschaft, Sozialwissenschaft) gefordert, neue Methoden, neue Verfahren, neue Verständnisse von Bildung zum gleichen Preis bereitzustellen, damit das Bildungspotenzial bzw. das Humankapital weiterhin den Erfolg garantieren kann.

- Zweitens: Wenn die Bildungspotenziale abnehmen und sich rückläufig entwickeln, die Pädagogik keine innovativen Ansätze zur Verfügung stellt, so werden zusätzliche Mittel kompensatorisch für den Bildungsprozess bereitgestellt; man hofft, dass sich durch veränderte finanzielle 
Rahmenbedingungen zwangsläufig ein bestimmter Bildungserfolg einstellt.

- Drittens: Sowohl die finanziellen Mittel als auch die innovativen pädagogischen Konzepte werden zur Verfügung gestellt, um Bildungspotenziale $\mathrm{zu}$ steigern und das wirtschaftliche Wachstum zu sichern.

Sailer, M. verweist darauf, dass es kaum einen Zusammenhang zwischen dem Bildungs- oder Lernprozess und der Produktivität gibt. Lediglich der formale Bildungsnachweis scheint bedeutsamer zu sein. Auch „die Screeningtheorie bezweifelt, dass der bloße Wissenserwerb zu höherem Einkommen verhilft. Somit wird der Produktivitätsthese der Humankapitaltheorie, nämlich das Investitionen in Bildung zu einer Steigerung der Produktivität führen und damit langfristige Erträge bewirken, eine Absage erteilt." (Sailer, M., S. 42 mit Verweis auf Timmermann 2002, S. 88)

Aus dieser Sicht heraus wäre die Abkehr von einem Wissensmanagement zu einem Bildungsmanagement verständlich. Nicht spezielles Fachwissen, sondern Persönlichkeitsmerkmale (Soft Skills) scheinen in ihrer Bedeutung besonders gewichtet.

Der dritte Weg prägt, insbesondere im Zusammenhang mit der Integration von Migranten, die Gegenwartsdiskussion: sowohl die Befähigung, die deutsche Sprache zu sprechen als auch die Kompetenz, nach deutschen Werten und Normen handeln zu können, werden als individuelle Bildungskategorien gefordert. Dabei wird die Deutsche Sprache nicht als eine fachliche Bildungskategorie angesehen, sondern sie ist ebenso wie personengebundene Einstellungen, Haltungen, Werte und Normen als Soft Skills den formalen Bildungskategorien zuzuordnen. Diese sollen in der deutschen Gesellschaft das Zusammenleben verbindlich prägen.

Welche Soft Skills bedeutsam werden, ergibt sich oft aus Zufälligkeiten. Sie entstehen vielfach aus dem Anspruch von Organisationen, Parteien, Verbänden und auch der Wissenschaften. Dabei zeigen sich Trends, rhythmische aber auch innovative Verläufe und Bewegungen.

So verweist Sailer (S.183 ff) in seiner Studie, in der er die Qualifikationsmerkmale in Stellenanzeigen zwischen 1950 und 2005 untersucht, auf eine interessante formale Kategorie, die in der Soft Skills Diskussion bisher nicht besonders beachtet wurde. Aus der Analyse von Stellenanzeigen konnte nachgewiesen werden, dass gegenwärtig neben den traditionellen Soft Skills die „Berufserfahrung als selektives Qualifikationsmerkmal“ offensichtlich immer bedeutsamer wird. 
In derselben Studie stellte Sailer (vgl. S. 214 ff) dar, „dass Arbeitgeber oft ein Idealbild vom Qualifikationsgefüge eines Bewerbers in Stellenanzeigen entwerfen." Dabei zeigt sich, dass sowohl personenbezogene als auch fachliche Bildungskategorien zu abstrakt und unverbindlich formuliert werden. Ein Grund hierzu liegt vielleicht in der Tatsache begründet, dass sich die Entwicklung des Potenzials eines Beschäftigten nur sehr schwierig prognostizieren lässt. Hier spielen Halbwertzeiten und veränderte Problemlagen in den betrieblichen Handlungsfeldern eine Rolle. Deshalb ist man oft auf der Suche nach formalen Bildungskategorien, die langfristig eine Eigendynamik entwickeln und sich als Indikatoren für die Entwicklung des Personals eignen. Diese Indikatoren sollen selbständig auf Lernprozesse, Tätigkeiten und Handlungsprozesse wirken und innovativ sein. Vielleicht ist man davon überzeugt, diese in der Handlungskompetenz gefunden zu haben. In der Diskussion über Soft Skills sind drei Ansätze bedeutsam:

\section{1) Die Pädagogische Strategie}

Bei knappen Mitteln sind die Bildungswissenschaften gefordert, das $\mathrm{Hu}-$ mankapital zu sichern. Die Wissenschaft soll möglichst kostenneutral neue Methoden, Verfahren und Theorien bereitstellen.

\section{2) Die finanzielle Strategie}

Die Bildungswissenschaft kann keinen innovativen Beitrag leisten. Finanzielle Investitionen der öffentlichen und privaten Institutionen in nicht bildungswissenschaftliche Bereiche sollen dem negativen Trend der Entwicklung der Bildungspotentiale entgegenwirken. So wird in materiale Objekte, neue Organisationsformen und formale Institutionen investiert; die finanziellen Mittel erreichen aber weder die Privatperson noch den Mitarbeiter im Betrieb. Der Einsatz der finanziellen Mittel präsentiert lediglich die politischen Proklamationen und hat formale Legitimation.

\section{Die Einheit von pädagogischer und finanzieller Strategie}

In diesen Fällen wird in die bildungswissenschaftlichen Prozesse finanziell eingegriffen, um die individuellen Bildungspotenziale und das wirtschaftliche Wachstum zu sichern. Die finanziellen Mittel sind auf die Adressaten der Bildung ausgerichtet. Der Bildungsprozess soll durch die Bildungswissenschaft effektiver gestaltet und nicht nur als bildungspolitische Floskel kommuniziert werden.

Die Diskussion um die Soft Skills und deren Bedeutsamkeit ist auch der Ausdruck fehlender bildungstheoretischer Erkenntnisprozesse. Selbst wenn man sich vom Zusammenspiel von pädagogischer und finanzieller 
Kompensation den positiven Effekt verspricht, Bildungspotenziale $\mathrm{zu}$ entwickeln, so muss der Bildungsbegriff jedoch vorab klar definiert und positioniert sein. Ehe Bildungspotenziale entwickelt werden, ist zunächst die Wesensstruktur des Phänomens Bildung zu bestimmen. Um Soft Skills verständlich und fassbar zu machen sollten sie einerseits inhaltlich so ausformuliert werden, dass das ihr zu Grunde liegende Bildungsverständnis verstehbar und nachvollziehbar wird. Die begriffliche Bestimmung der Soft Skills darf sich weder von den Verhaltenswissenschaften (z.B. Psychologie) noch von den informationstheoretisch orientierten Kommunikationswissenschaften dominieren lassen, sondern ist als eine Bildungsvariante darzustellen. Bildung führt den Menschen zur Freiheit und zur Autonomie. Bildung, und damit auch die personengebundenen Soft Skills, sind keine Techniken, sondern Teil der Persönlichkeit, die langfristig den wirtschaftlichen und persönlichen Erfolg sichern können. Eine einseitige Diskussion um Soft Skills und ihre bildungstheoretische Isolierung verweisen auf Positionen wie

- Investitionen in Bildung haben keinen nachhaltigen Effekt auf die Produktivität in Unternehmungen,

- formale Bildungsnachweise sind effektiver,

- die Produktivitätsthese des Humankapitals ist $\mathrm{zu}$ falsifizieren (vgl. Sailer, M., S. 42; Timmermann 2002 S. 881)

Obwohl die Bedeutsamkeit von Soft Skills bei der Rekrutierung von Personal unstrittig zu sein scheint, zeigen sie sich aber als personenbezogene und fachliche Bildungskategorien als zu abstrakt formuliert und bleiben deshalb ineffektiv. Die Soft Skills müssen jedoch vom Handlungsfeld (Praxis) her gesehen, verstanden und formuliert werden. Sailer (2009, S.183 ff) konnte nachweisen, dass neben den traditionellen Soft Skills die „Berufserfahrung als selektives Qualifikationsmerkmal in Stellenanzeigen“ eine markante Bedeutung bekommt. Es schwingt bei den Vorstellungen über Soft Skills auch immer etwas Praxis mit, ohne diese jedoch konkret zu benennen. Aber auch in diesen Fällen bleibt die "Praxis“ als eine mögliche Soft Skills Variante im Allgemeinen stecken, ohne seinen Adressaten differenzierte Auskunft über konkrete Inhalte zu geben.

Es scheint die berufliche Handlungskompetenz zu sein, die als übergeordnete Kategorie betrieblicher Bildung, die fachliche Bildung und die personengebundenen Spezifika der Soft Skills verknüpft. Wenn sich diese Annahme bestätigen lässt, so könnte die berufliche Handlungskompetenz als Indikator für erfolgrei- 
ches Tun angesehen werden. Um wirken zu können muss die berufliche Handlungskompetenz dann aber als Spezifikum differenziert herausgearbeitet werden und darf nicht für die verschiedensten Handlungsfelder gelten.

Soft Skills haben für Maßnahmen der Personalauswahl, der Personalentwicklung und der Personalbewirtschaftung an Bedeutung gewonnen. Soft Skills sind wesentlicher Bestandteil des Humankapitals. Sie werden in weiterführende Diskussionen zum Wissens-, Kompetenz- und Bildungsmanagement eingebunden. Während durch fachliche Kompetenzen operative Abläufe betrieblicher Prozesse (Arbeit, Tätigkeiten, Verhalten) erfolgreich gestaltet werden können, sind Innovationen und kreative Prozesse insbesondere von Soft Skills, den formalen Bildungskategorien, abhängig. Hierzu zählen z.B. nicht nur die soziale- und die kommunikative Kompetenz, sondern immer mehr auch die Fähigkeiten zum Sinn- und erklärendem Verstehen und in verschiedenen Zusammenhängen systematisch und differenziert denken und handeln zu können. Spezielle Soft Skills reduzieren sich dagegen zu Kategorien fachlicher Kompetenz. Dies trifft z.B in den Fällen zu, wenn zwar allgemeine sprachliche Fähigkeiten (Deutsch, Englisch) gefordert sind, diese sprachlichen Fähigkeiten aber für ein spezielles Handlungsfeld gelten sollen.

\section{Die Unternehmens-, Verbands- oder Schulkulturen als sinnstiftende Wirklichkeit für Soft Skills}

\section{Soft Skills als Ausdruck von Vorstellungen und Theorien}

Soft Skills bekommen erst dann einen Sinn, wenn sie sich z.B. über Unternehmens-, Verbands- oder Schulkulturen begründen und rechtfertigen lassen. Sie haben als Bildungskategorie auch nur eine relative Legitimation und Bedeutsamkeit.

Wo finden wir aber die Legitimation für die auf die betriebliche Praxis bezogenen Soft Skills? Wir müssen Sie aus dem Kontext der betrieblichen Wirklichkeit erschließen. Sie sollten weder aus dem politischen, dem gesellschaftlichen noch dem privaten Kontext allgemein und übergreifend abgeleitet und bestimmt werden, sondern sich aus der Unternehmenskultur und den rationalen Ansprüchen betrieblicher Praxis ergeben. Soft Skills sind nicht nur über verbale Werbekampagnen zu deklarieren, sondern sie müssen sich konkret auf den Ablauf betrieblichen Handelns beziehen. Um den Soft Skills Sinn zu geben damit sie für den 
Betrieb sinnvoll sind, müssen sie sich aus einer Unternehmenskultur begründen und als Prinzipien des Sollens bestimmt und vermittelt werden. Sie stehen nicht alleine für sich, sondern sie müssen sich widerspruchsfrei zu den anderen Kategorien der Unternehmenskultur verhalten. Insbesondere die materiellen Anforderungen, die definierten Tätigkeitsfelder in der Praxis und nicht zuletzt die Vorstellungen von den Mitarbeitern spielen eine bedeutsame Rolle.

Soft Skills sind immer Ausdruck spezifischer, differenzierter Vorstellungen und Entwürfe:

(1) Wirtschaftswissenschaftlich orientierte Soft Skills

- Für Ziele sorgen

- Organisieren

- Entscheiden

- Kontrollieren

- Menschen entwickeln und Fördern

- Vertrauen schaffen

- Ganzheitliches Denken

- Die beruflich handelnde Person vereinigt sowohl Fachliche Kompetenz als auch Soft Skills.

- Didaktische Kompetenz (vgl. Malik, F.: Führen, Leisten, Leben. München 2001)

(2) Gesellschaftspolitisch orientierte Soft Skills

Sowohl in der gegenwärtigen Diskussion über den Zuzug von Fachkräften für die Wirtschaft als auch in der Diskussion über die Integration von Migranten werden insbesondere gefordert:

- Handlungskompetenz

- Sprachliche Kompetenz

- Soziale Kompetenz

- Kulturale Kompetenz

- Politische Kompetenz

- Medienkompetenz

Eine zentrale Position nimmt dabei die Handlungskompetenz ein. Sowohl die kulturale- als auch die soziale Kompetenz sind dafür Legitimationskategorien. Die sprachliche Kompetenz, als funktionale Bildungskategorie der Handlungskompetenz, beeinflusst entscheidend den Erfolg bzw. Misserfolg in den Handlungsfeldern. Aus diesem Grund bekommt die 
Sprachliche Kompetenz zu Recht in der Bildungsdiskussion einen besonderen Stellenwert.

(3) Psychologisch orientierte Soft Skills

- Konfliktfähigkeit

- Problemlösungsfähigkeit

- Interaktionsfähigkeit

- Emotionale Intelligenz (EQ)

- Beziehungsfähigkeit

- Sich und andere motivieren können

(4) Soft Skills als Pädagogische Kategorien

Bildung, Erziehung, Lernen, Sozialisation, Wissen, Kompetenzen, Fähigkeiten, Fertigkeiten, Haltungen, Gesinnungen sind pädagogische Kategorien, deren theoretisches Verständnis einen Beitrag leisten, um Soft Skills inhaltlich und didaktisch aufzubereiten. Die Diskussion wird im pädagogischen Kontext sehr abstrakt geführt und verweist auf die Bereiche der Lernenden und der Lehrenden.

Pädagogische Soft Skills

a) auf die Lernenden bezogen

- Sozialkompetenz

- Kommunikative Kompetenz

- Medienkompetenz

- Methodenkompetenz

- Lernkompetenz

- Sprachkompetenz (Deutsch, Englisch)

b) auf die Lehrenden bezogen

- Sozialkompetenz

- Kommunikative Kompetenz

- Medienkompetenz

- Methodenkompetenz

- Didaktische Kompetenz

- Kompetenz zu motivieren

- Vermittlungskompetenz

- Handlungskompetenz 
Auf einigen Ebenen ist der Abstraktionsgrad so hoch (vgl. Sozialkompetenz, Kommunikative Kompetenz), dass zwischen Lernenden und Lehrenden inhaltlich kaum unterschieden wird. Teilweise reduzieren sich die Ausführungen zu Floskeln und Wortbenennungen. Eine Wesensschau der begrifflichen Struktur ist oft nicht durchführbar. Es zeigt sich ein hermeneutischer Bankrott. Aus der nachfolgenden Grafik wird deutlich, dass die Wirkungen von Soft Skills auf die Handlungsfelder Arbeit, Familie oder Freizeit nicht zufällig in Erscheinung treten, sondern sich auf Grund des Einflusses von Kultur und Gesellschaft über die Pädagogik zwangsläufig bedingen.

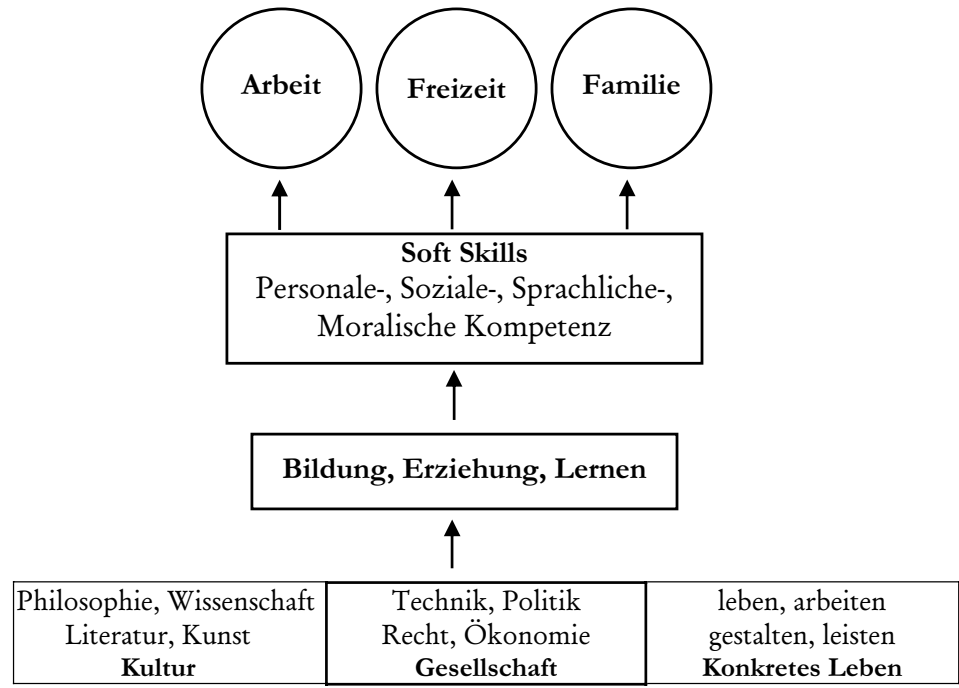

Abb.: Soft Skills im Einfluss von Kultur und Gesellschaft

II.

\section{Die anthropologische Dimension von Soft Skills}

Es ist davon auszugehen, dass sich sowohl das Verständnis von Lernen, Bildung und auch von Soft Skills von einer anthropologischen Grundlage begründet. Somit ist die These zulässig, dass 
- differenzierende anthropologische Sichtweisen nicht nur zu einem unterschiedlichen Verständnis von Lernen, Bildung und Soft Skill führen, sondern auch konkret auf die inhaltliche Struktur und die Funktionalität dieser pädagogischen Begriffe Einfluss nehmen.

Der Mensch ist Ausdruck kultureller und zivilisatorischer Annahmen. Im kulturellen Bereich sind es sowohl philosophische-, religiöse- als auch künstlerische Dispositionen; im zivilisatorischen Bereich finden wir oft ökonomische, politische und auch technische Grundannahmen.

Der Mensch als Ausdruck kultureller und zivilisatorischer Annahmen

\begin{tabular}{|l|l|}
\hline \multicolumn{1}{|c|}{ Kulturell } & \multicolumn{1}{c|}{ Zivilisatorisch } \\
\hline philosophisch & ökonomisch \\
\hline religiös & politisch \\
\hline künstlerisch & technisch \\
\hline
\end{tabular}

Kulturelle und zivilisatorische Bedingungen prägen das Verständnis vom Menschen. Dabei zeigen sich sowohl Vorstellungen, die sich mit Idealtypen, den einzelnen Wissenschaften oder dem praktischen Leben in Verbindung bringen lassen. Bei den Idealtypen gibt es Bezugspunkte zum homo sapiens, homo ludens, homo discens, homo politicus, homo soziologicus, homo faber oder dem homo oeconomicus. Für anthropologische Begründungen aus dem Kontext der Wissenschaften positionieren sich insbesondere die Psychologie, die Soziologie, die Ökonomie; aber auch die Pädagogik. Als sehr einflussreich zeigen sich Wirkungsmechanismen, die sich vom homo oeconomicus her verstehen: Wie muss der Mensch denken und verstehen, damit er ökonomisch handeln kann? Wie denkt und versteht ein Mitarbeiter, wenn er nicht ökonomisch-rational ausgerichtet ist? 
Warum benötigen Wirtschaftsunternehmen Menschen, die nicht den Gesetzen einer ökonomischen Rationalität folgen sollen?

Die anthropologisch determinierten Soft Skills sind personengebunden; Einzelnen, Gruppen, oder den Menschen in spezifischen Organisationen wie z.B. Betrieben, Schulen, Verwaltungen oder Verbänden zugeordnet.

Größere Bedeutungen für betriebliche Vorstellungen von einem Mitarbeiter ergeben sich aus der Sicht der betrieblichen Handlungsfelder:

- Welche beruflichen Ansprüche werden wo, wann und wie gestellt?

- Welche Ansprüche werden für welche Handlungsfelder an das Personal gestellt?

- Welche Ansprüche stellt die Personalentwicklung?

Die methodisch-didaktische Konzeptionierung der Soft Skills ist eine Aufgabenstellung für die Pädagogik, wobei sich ihre eigene anthropologische Positionierung, dass der Mensch auf Lernen, Bildung und somit auch auf Soft Skills verwiesen ist, immer aus dem Verständnis anderer Wissenschaften, wie der Biologie, der Soziologie, der Philosophie bzw. den Wirtschaftswissenschaften zu erklären und zu verstehen ist. Bildung, Erziehung, Lernen und Sozialisation werden erst aus dem Blick anderer Wissenschaften bzw. konkreter Praxisformulierungen inhaltlich konkret fassbar. Die anthropologische Position aus dem Kulturverständnis eindeutig darzustellen, ist für viele Unternehmungen, Bildungsinstitutionen, Parteien oder Verbände nicht nur schwierig, sondern äußerst problematisch und konfliktträchtig. Ein ausgewiesenes, transparentes Verständnis vom Mitarbeiter hat zu viele Angriffsflächen für divergierende Positionen in betrieblichen Gemeinschaften.

Aber auch das praktische Leben prägt die anthropologischen Vorstellungen und nimmt Einfluss über Eltern, Erzieher aber auch über die VIPs, den sog. Persönlichkeiten des öffentlichen Lebens. Dies alles betrifft nicht nur den Menschen in seiner Ganzheit, sondern auch bezüglich bestimmter Ausschnitte seiner Person, z.B. den Soft Skills. Es wird anthropologisch gesehen dann kompliziert, wenn der Mensch als Mitarbeiter in einem Betrieb keinem einheitlichen Denkansatz unterliegt, sondern aus einem widersprüchlichen Puzzle zusammengesetzt wird. Das ist dann eine besondere Problemlage, wenn man sinnvolle Ansprüche an Mitarbeiter formulieren und kommunizieren will, aber auf Grund der inneren Widersprüchlichkeit, sich Sinnlosigkeit und Misserfolg zwangsläufig ergeben. 
Soft Skills verstehen und erklären sich von einem zu Grunde gelegten Menschenbild her. Dies kann formal begründet und hergeleitet sein; es kann sich aber auch unreflektiert aus dem jeweiligen Lebens- und Handlungsfeld eines Betriebes, einer Schule, einer Familie oder z.B. einer Partei ergeben.

So können sich z.B. Soft Skills aus einem Verständnis über den

- homo öconomicus,

- homo soziologicus,

- homo faber,

- homo educandus,

- homo discens,

präsentieren und definieren. In diesem Zusammenhang sei darauf hingewiesen, dass der Umgang mit der Begrifflichkeit „lernende Organisationen“ nicht korrekt ist, da es immer Menschen sind, die lernen. Organisationen können nicht lernen, denn sie sind nur Ausdruck der lernenden Subjekte. Dies wurde jedoch in den Sozial- und Wirtschaftswissenschaften in jüngster Zeit oftmals nicht immer korrekt nachvollzogen. Insbesondere im Zusammenhang mit der Wissensgesellschaft. Hier zeigte sich insbesondere auch der Einfluss der Arbeits- und Organisationspsychologie, die nicht von einem pädagogisch diskutierten Bildungsverständnis ausging bzw. theoretisch nicht in der Lage war, von einem solchen auszugehen.

\section{II.}

\section{Die materiale, sachliche Dimension von Soft Skills}

Es wird oft das Argument vorgetragen, der Wille sei vorhanden, die fachliche Befähigung fehlt; oder man kennt seine Werte und Normen, nach denen man sich ausrichten soll und ausrichten will und deshalb fähig ist, in spezifischen Situationen moralisch handeln zu können. Das ist ungefähr so, als würde man Menschen im Erziehungs- oder Sozialisationsprozess lediglich Wissen bzw. Informationen über Werte und Normen vermitteln, jedoch keine praktischen Möglichkeit bieten zu lernen, nach bestimmten Grundsätzen handeln zu können. Soft Skills sind zwar sehr wesentlich auch von ihrer inhaltlichen Ausformulierung bestimmt, ihre ausschließliche Reduktion darauf macht sie jedoch in wesentlichen Teilen bedeutungslos. Sie tendieren dann zu einem funktionalen, in die fachliche Richtung weisenden Bildungsverständnis und sind sehr kurzen 
Halbwertzeiten ausgesetzt. Auf der Ebene der materialen/sachlichen Dimension von Soft Skills ist der pädagogisch/didaktische Anspruch besonders gefordert. Die Soft Skills sind für den Einzelnen lerntheoretisch so aufzubereiten, dass mit Erfolg die pädagogischen Zielsetzungen erreicht und die praxisorientierten Handlungsabläufe entsprechend gestaltet werden können.

II.

\section{Die ethische Dimension (Werte/Normen) von Soft Skills}

Der Sinn von Soft Skills bestimmt sich nicht nur über die materialisierten, inhaltlichen Ausformulierungen, sondern auch über die ethische Dimension, den Werten und Normen. Diese sind in der Unternehmens-, Verbands- oder Schulkultur für alle Kulturkategorien leitend und bestimmend; nicht nur im einzelnen bezüglich des anthropologischen Grundgedankens über den Mitarbeiter, den Lernenden, sondern auch für die Auswahl und Rechtfertigung von Sachen, Gegenständen, Tools und Soft Skills. Alle Kulturkategorien verhalten sich untereinander interdependent, sie sind gegenseitig abhängig. In diese innere Rationalität und Ethik sind die Soft Skills eingebunden. Sie sind Teil eines Ganzen. Sollen Soft Skills sinnvoll sein, so müssen sie sich von einer bestimmten Ethik her bestimmen und zuordnen lassen.

Aus diesem Zusammenhang heraus wäre verstehbar, dass z.B.

- Verantwortung

- Ehrlichkeit

- Zufriedenheit

- Leistungsfähigkeit/Leistungsbereitschaft

- Berufserfahrung/Praxiserfahrung)

- Motivation

erst dann Sinn machen, wenn sie nicht nur konkret ausformuliert, sondern auch in einen Werte- und Normenkontext gesetzt werden. Es stellt sich nicht nur die Frage nach konkretem verantwortlichem Handeln, sondern auch nach dem Grund, warum, weshalb und weswegen es bedeutsam und wertvoll ist, eine spezielle Verantwortung übernehmen zu sollen und zu wollen. Was für die einen verantwortlich ist, kann für andere Mitmenschen unverantwortlich sein. Es genügt nicht, lediglich die Ausführung von Verantwortung zu lernen, sondern mit einem verantwortlichem Handeln sollte auch immer die Einstellung und Gesin- 
nung des handelnden Subjekts verknüpft werden.. Erst dann lässt sich eine verantwortliche Tätigkeit auch moralisch vom Handelnden tragen. Löst man die Soft Skills jedoch aus dem Kontext von Ethik (Wert- und Normensystem) Ethos (Gesinnung und Haltung des Subjekts) und der Moral (bewertetes Handeln), dann reduzieren wir sie auf funktionelles Verhalten. Deshalb lassen sich Soft Skills auch nicht trainieren, sondern sie sollten immer Ausdruck eines gebildeten, selbständig handelnden Menschen sein. Erziehung und Sozialisation nehmen als Lernprozess einen entscheidenden Einfluss auf die Entwicklung der Persönlichkeit. Aus dem praktischen Leben heraus, den konkreten Handlungsbereichen der tätigen Mitarbeiter, formen sich die Soft Skills als Kategorien der Erziehung und der Sozialisation.

\section{II.}

\section{Sinnvolles Tun als Ausdruck von Soft Skills}

In der Unternehmenskultur werden Ansprüche an den Menschen, an seine Wertund normorientierten Haltungen und Einstellungen (Ethik, Ethos, Moral) präsent und die unternehmerischen Vorgaben (Vorstellungen, Ideen, Theorien) über die materiellen und inhaltlichen Bestimmungen der in der Praxis einzubringenden Tätigkeiten. In den anthropologischen Bestimmungen von Soft Skills zeigen sich Sollensprinzipien, die die Unternehmenskultur prägen. Diese Vorstellungen von Mitarbeitern in den Unternehmungen geben z.B. den Konzepten der Personalentwicklung den entsprechenden Sinn und bilden die Grundlage für eine sinnvolle (erfolgreiche) Unternehmensführung.

Aus den Unternehmenskulturen lassen sich empirisch anthropologische Positionen und Sichtweisen ableiten, deren Sinn oft nicht in den ökonomischen Prinzipien des jeweiligen Unternehmens selbst zu finden ist, sondern lediglich durch politisch oder soziologisch determinierte Marketingstrategien populistisch gesetzt wird. Teilweise geschieht dies, um die gewinnorientierten Absichten zu verschleiern. Somit lassen sich oft aus den Unternehmenskulturen widersprechende anthropologische Sinnsetzungen herausarbeiten. Es zeigen sich vielfach sowohl gelebte als auch proklamierte Vorstellungen von Mitarbeitern. Solch ein Sinnwiderspruch führt oft, sowohl auf unternehmerischer als auch auf Kundenseite, zu Sinnverlusten, weil der Bedeutungszusammenhang nicht mehr zweifelsfrei und eindeutig ist. Diese Tendenz zeigt sich u.a. auch in der Diskussion um den Sinn von Soft Skills in den personalpolitischen Entscheidungen: Entscheidet 
man sich für Persönlichkeitsfaktoren und definiert den Anspruch an den Mitarbeiter aus der notwendigen Sicht des Unternehmens, oder folgt man dem politischen Mainstream. Um aus diesem Widerspruch herauszukommen, entscheidet man sich für Soft Skills auf einem sehr hohen Abstraktionsniveau.

Betriebliche Wirklichkeit als Sinnstruktur

(sinnbezogene Führung)

Die Wirklichkeit des

Tuns

(ich tue es)
Unternehmenskultur als Wirklichkeit

(Prinzipien des Sollens)

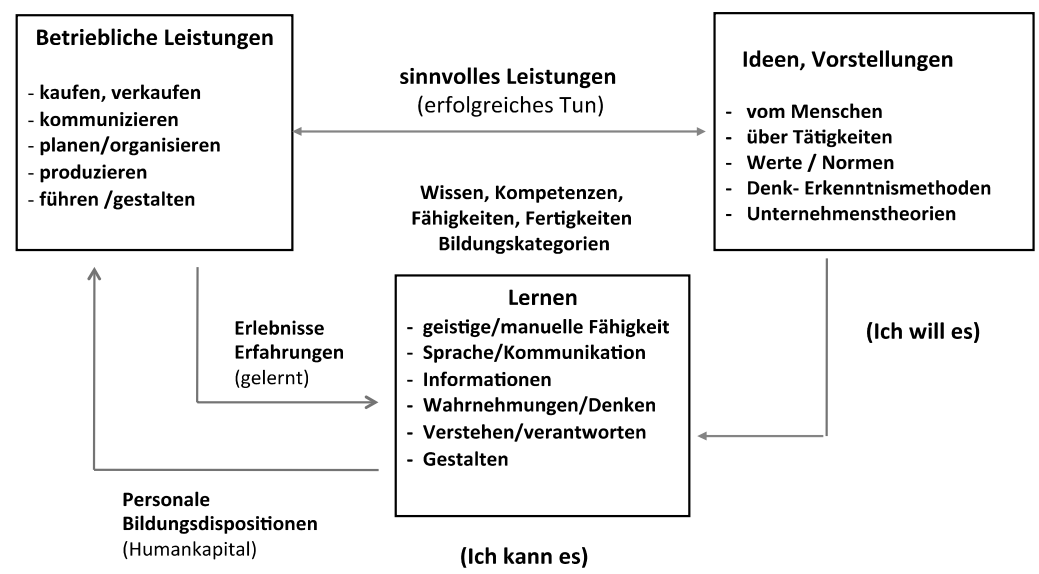

Abb. Die Sinnstruktur von Bildung

Folgt man der Grafik über die „Sinnstruktur von Bildung“ so könnte man meinen, um Erfolg zu haben genügt es zu lernen, wie das operative Geschäft betrieblichen Handelns zu gestalten ist.

Auf der Wirklichkeitsebene des „Tuns“ sind in der Praxis aber auch eindeutig personenbezogene, nicht fachliche Fähigkeiten, wie soziales oder kommunikatives Grundverhalten gefordert; oder Haltungen und Einstellungen, als Ausdruck von Ethik, Ethos oder Moral, um der Handlungsebene Sinn zu geben und sie langfristig erfolgreich $\mathrm{zu}$ gestalten. Es ist darauf $\mathrm{zu}$ achten, und darauf zielt die Verantwortung unternehmerischer Führung ab, die Einheit der drei betrieblichen Wirklichkeitsebenen

zu gewährleisten. Die personenbezogenen Soft Skills haben eine wert- und normenorientierte Bestimmung des „Sollens“, aus der sich das „Wollen“ einzelner 
Entscheidungsträger ableiten und legitimieren lässt. Über die personenbezogenen Soft Skills werden auf der handlungsbezogenen Ebene betrieblichen Tuns die Determinierungen des Sollens mit den subjektiven Haltungen des Wollens über den Mitarbeiter selbst verknüpft.

Der Mitarbeiter folgt hier einem sinnvollen und erfolgreichen Grundprinzip:

- Ich will, was ich soll und ich tue, was ich kann.

Soft Skills, als Einstellungen und Haltungen, müssen gelernt werden, wie jede fachliche Fähigkeit. Dieser Lernprozess ist sozialisationsbedingt und lässt sich nicht durch kurzfristig angesetzte Lehrveranstaltungen didaktisch umsetzen, sondern unterliegt einem besonderen methodischen Anspruch. Da sich Soft Skills auf Handlungsabläufe auszurichten haben, sie der Handlungskompetenz zuzuordnen sind, lassen sie sich auch nur über handlungsorientierte Lernmethoden vermitteln. Dies gilt insbesondere für Soft Skills, die Einstellungen, Haltungen und Gesinnungen bestimmen. Personenbezogene Soft Skills, die sich mehr oder weniger über das Verhalten, Techniken, Wissen definieren, ist die Gestaltung von Lernprozessen kurzfristig machbarer.

Das Sollen bestimmt unsere Tätigkeiten, unser Können und unser Tun. Unser Ethos verweist auf der Ebene des Könnens nicht nur auf Haltungen und Einstellungen einer Person, die sich von der Werteebene herleiten und das Wollen bestimmen, sondern unser Ethos ist auch geprägt von Einstellungen und Haltungen im Umgang mit Normen, Regeln, Gesetzen, Geboten und Verboten.

Der Mensch ist auf Entfaltung ausgerichtet und braucht einen Fixpunkt, ein Ziel, das ihm seine Tätigkeit als sinnvoll verstehen lässt.

Der Mensch benötigt aber auch Beschränkungen durch Regeln und Normen. Dies gibt ihm Sicherheit für sein Handeln, damit er sich nicht in seinen Aktivitäten verliert.

Der beruflich tätige Mensch steht nicht nur in der Spannung zwischen Sollen, Können und Tun, sondern seine Tätigkeiten geben erst dann einen Sinnzusammenhang, wenn die Interdependenz zwischen Sollen, Können und Tun widerspruchsfrei bleibt.

Unsere Leistungen im Betrieb oder in der Schule werden erst über eine Begründung (Legitimation) sinnvoll und nützlich.

Die Legitimation und die Begründung für die Leistungen finden wir in den Vorstellungen über Leistungen von Betrieben, Organisationen oder Schulen. 
In ihren anthropologischen Begründungen werden Soft Skills zu Prinzipien des Sollen und des Wollens. Sie bleiben aber oft in Haltungen und Gesinnungen stecken und haben als Ethos für die Praxis kaum Relevanz, da ihren Trägern für die Umsetzung die nötige sachliche/materiale/inhaltliche Befähigung fehlt.

\section{Zur Dialektik von Soft Skills und Fachlicher Bildung Soft Skills in der Spannung zwischen Materialer und Formaler Bildung}

Lernfähigkeit, Lernwilligkeit, Lernbereitschaft als personengebundene, formale Bildungskategorien, stehen in der gegenwärtigen Diskussion weniger im Kontext des Lernens aus einem pädagogisch/didaktischen Verständnis heraus, das sich inhaltlich/sachlich orientiert (fachliche Kompetenz, fachliche Bildung), sondern sie stehen vorwiegend im Kontext eines prozessualen/methodischen Verständnisses der Psychologie.

Eine Antwort auf die Frage nach den Bildungsinhalten stellt sich für die Psychologen nicht. Was gelernt wird ist nicht die entscheidende Prämisse. Wie gelernt werden soll und was die lernende Person an persönlichen und methodischen Fähigkeiten zur Optimierung ihres eigenen Lernprozesses einbringen kann steht zur Disposition.

In den Mittelpunkt des Interesses rückt der Prozess des Lernens. Hier zeigt sich eine Dominanz der Konstruktivistischen Lerntheoretiker, die Lernen als KoKonstruktion, als einen sozialen Prozess verstehen. Die Bedeutsamkeit dessen, was in diesem Prozess an Fähigkeiten, Fertigkeiten und Kompetenzen vermittelt wird, bestimmt sich nicht von objektiven Bildungsinhalten her, sondern von der individuellen Ausgangslage des Lernenden und seiner kontextualen Bestimmungen. Das Ziel dieses Ansatzes ist es, den Menschen für das Leben zu befähigen. Dies geht eindeutig zu Lasten objektiver Bildungsstandards der Disziplinen, Fächer, Arbeits- und betrieblicher Handlungsfelder. In der Pädagogik ist dieses Verständnis bei den Vertretern des Portfolio-Ansatzes zu finden. Die individuelle Lernbiografie, im Portfolio des einzelnen Lerners dargestellt und beschrieben, präsentiert die individuelle Lernleistung, die sich aber objektiv nicht als Leistung vergleichen lässt. Lernen negiert hierbei objektive, kulturale und fachliche Bildungsstandards. Wenn sich die einseitige Ausrichtung auf die Vermittlung von 
Soft Skills bezieht, den individuellen Befähigungen im Umgang mit Lernmethoden, dann verbleiben wir im Zustand der „Halbbildung“.

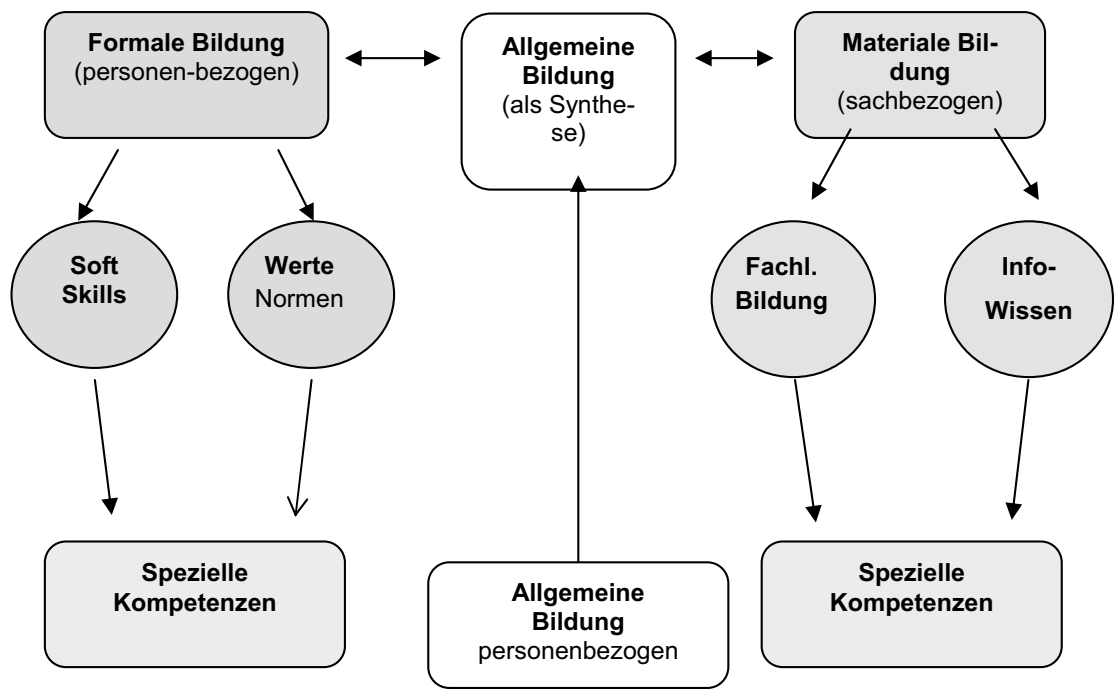

Abb. Die bildungstheoretische Einordnung von Soft Skills

\section{III.}

\section{Soft Skills als berufliche Handlungskompetenz}

In der Diskussion über Allgemeine Bildung nimmt die personenbezogene Kategorie der Handlungskompetenz eine bedeutende Stellung ein. Die Handlungskompetenz wird zum Ziel der Auseinandersetzung in den verschiedensten Handlungsfeldern. Es ist die Praxis, das operative Geschäft, dass sich Fragen nach Erfolg und Misserfolg in immer größerer Herausforderung stellen. Was und womit haben wir den größten Erfolg? Dabei bekommt die Ressource Mensch eine immer größere Bedeutung und die Kategorie Bildung verfolgt die Personalentwickler in den Betrieben. Dabei genügt es aber nicht, dass der zukünftige Mitarbeiter umfassend und vielfältig mit Kompetenzen und Wissen ausgestattet ist, sondern er muss möglichst so ausgebildet und befähigt sein, dass er direkt in den Arbeitsprozess eingegliedert werden kann. Dahinter verbirgt sich die Forderung nach einer beruflichen Handlungskompetenz. Sie ist die Synthese aus Soft Skills und 
fachlicher Bildung und das Ergebnis eines dialektischen Prozesses zwischen Fachkompetenz einerseits und Sozial- und Selbstkompetenz andererseits. Es ist die aus Kenntnissen, Wissen, Fähigkeiten, Fertigkeiten aber auch aus Einstellungen, Haltungen und Gesinnungen gebildete Vermögen einer Person, betriebliche Herausforderungen kompetent zu bewältigen.

Die Verlagerung des Erwerbs dieser Praxiskompetenz zeigt sich nicht nur im schulischen, sondern sehr eklatant auch im universitären Bereich, wenn es um die Gestaltung von Bachelorstudiengängen geht.

Die Handlungskompetenz integriert Bildungskategorien wie die der Fachkompetenz, Selbst-, Sozial-, Methoden- oder Lernkompetenz und insbesondere immer mehr auch die sprachliche Kompetenz:

(1) Fachkompetenz

Das operative Geschäft inhaltlich und sachlich zu kennen.

(2) Selbstkompetenz

Die eigenen Haltungen erfolgsorientiert und leistungsbezogen einzusetzen. Private und betriebliche Werte und Normen nicht als Widerspruch zu verstehen.

(3) Sozialkompetenz

Sich auf der Beziehungsebene gem. den Anforderungen der operativen Prozesse zu präsentieren.

(4) Methodenkompetenz

Bestimmte Arbeitsabläufe zielgerichtet planen zu können.

(5) Lernkompetenz

Fachartikel, Anordnungen und Veränderungen zu kennen und zeitgemäß auf ihre Anwendbarkeit in den Betriebsabläufen zu überprüfen. Sich den neuen betrieblichen Herausforderungen zu stellen.

(6) Die Kommunikative Kompetenz (sprachliche Kompetenz) als Sonderfall Sie könnte einerseits Soft Skills darstellen, wenn es um das allgemeine Verstehen von formalen, verbalen sowie nonverbalen Formen und Symbolen geht. Aber andererseits könnte die Kommunikative Kompetenz auch der fachlichen Kompetenz zugeordnet werden, wenn es um die konkrete Gestaltung von Betriebsabläufen geht. Z.B. wer definiert und vereinbart wann, mit wem und für wen betriebliche Ziele. Oder wer evaluiert, dokumentiert und präsentiert Projektabläufe, wie und für wen? Oder wie gestalte ich die Gebrauchsanweisung meiner Produkte. 
In der gegenwärtigen Diskussion über den Zuzug von Fachkräften für die Wirtschaft wird die Handlungskompetenz oft als

- Synthese von Fachkompetenz, Sprachkompetenz und kulturaler Kompetenz

verstanden und gefordert.

Hierbei nimmt insbesondere die kulturale Kompetenz, und durch sie bedingt die Sprachkompetenz, einen bedeutenden Stellenwert ein. Sie war unterschwellig immer als Herausforderung gegeben, aber niemals so eindeutig deklariert worden wie in der gegenwärtigen Situation.

Der Trend und der Anspruch in der Personalentwicklung setzt einen eindeutigen Akzent auf Soft Skills. Das zeigt, dass man zwar für den optimalen Ablauf in den Betrieben die fachliche Kompetenz als etwas Selbstverständliches ansieht, aber immer eindeutiger die personalen Kompetenzen fordert, die sich sehr kulturund wertabhängig determinieren. Man scheint der Ansicht zu sein, dass gerade diese personalen Kompetenzen besonders geeignet sind, einen erfolgreichen und möglichst konfliktfreien Arbeitsablauf zu gewährleisten. Das Ziel der Personalentwickler scheint die berufliche Handlungskompetenz zu sein. Nach Ryschka u.a.(S. 20) ist es das Vermögen einer Person, die beruflichen Herausforderungen selbstorganisiert zu bewältigen. Dieses Vermögen setzt sich aus Kenntnissen, Fertigkeiten, Fähigkeiten, Einstellungen und Gesinnungen zusammen.

Auf der Wirklichkeitsebene des Tuns präsentert sich der Mitarbeiter über seine betrieblichen Aktivitäten und Leistungen, den Arbeitsabläufen. Ein Mitarbeiter muss

- handeln, führen, gestalten,

- planen, einkaufen, verkaufen,

- kontrollieren kommunizieren, reflektieren,

- ausbilden, qualifizieren, produzieren,

- beurteilen, bewerten, begutachten.

Die berufliche Handlungskompetenz erfordert Soft Skills verschiedenster Art. Diese werden in den Handlungsfeldern konkret und praxisrelevant. Erst über das Handeln selbst werden sie sinnvoll. Soft Skills und die fachliche Kompetenz einer Person bestimmen den Erfolg bzw. Misserfolg bei der Lösung von Problemen bzw. bei der Durchführung von Arbeitsaufträgen. Wie aus der nachfolgenden Abb. sichtbar, vereinigt die beruflich handelnde Person sowohl Fachliche Kompetenz als auch Soft Skills. 


\section{Der Arbeitsauftrag als Ausgangslage des Lernens}

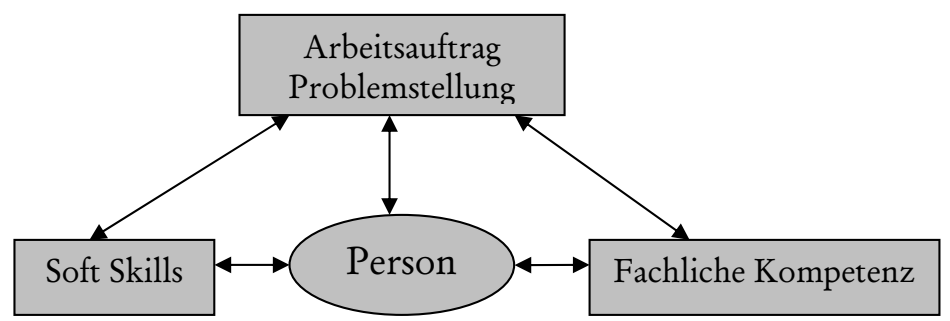

Malik hat in seiner Führungslehre darauf verwiesen, dass z.B. die Verantwortung bei dem Individuum liegen muss, dass sich aber konkret immer die Frage stellt, "was diese Person wissen und können muss und welche Kompetenzen sie braucht, um die Verantwortung auch tatsächlich übernehmen zu können. Dies ist die praktisch wirksame Art, Organisationen zu bewegen und zu führen, und nicht etwa die abstrakte Bestimmung von Aufgaben-, Kompetenz- und Verantwortungspaketen, also die Stellenbildung. Diese ist zwar auch wichtig, aber Stellen sind nicht das, was eine Organisation bewegt und steuert.“ (Malik, F. S. 224) Auf einen ähnlichen Zusammenhang verweist Malik, in dem er die Etablierung von Feedback (personengebundene Fähigkeit von Führungskräften) als eine Chance sieht, da wirksame Manager einer abstrakten Kommunikation zu tiefst misstrauen : „Sie gehen hin und schauen und reden mit den Leuten; sie wollen die Dinge mit eigenen Augen sehen und wenn möglich, mit ihren Händen greifen. Damit verschaffen sie sich über die Zeit ein Maß an Sachkenntnis und Vertrautheit mit der Situation, das durch nichts anderes erreicht werden kann.“ (Malik, S. 225)

Hier lernt eine Führungskraft aus sich heraus sachliche Fähigkeiten, weil sie in der Lage ist, Feedback als Soft Skills strategisch einzusetzen. Die Führungskraft muss aber über ein bestimmtes Ausmaß an fachlicher Grundbildung verfügen, damit es zum fachlich orientierten Lernfortschritt kommt. Fehlt dieses fachliche Bildungsfundament, so verfängt sich jedes Feedback an einer emotionalen Oberflächlichkeit oder in der Sackgasse von „gut“ oder „hat mir nicht gefallen“. Dann steht das „persönliche Empfinden“ oder eine „subjektive und nicht nachvollziehbare Einstellung“ bis hin zu dem sog. „Bauchgefühl“ der Sache, dem Inhalt gegenüber. 
Wenn diese Feedback determinierten Soft Skills nicht den Weg zur fachlichen Befähigung finden, so bleiben sie formal und abstrakt.

Erwähnenswert hierzu sind die Versuche zum fachlich orientierten Feedback, oder die formalisierten und abstrakten Abläufe von Metakommunikation in den Seminaren einer Universität. Wie kann z.B. ein Student im 1. Semester zur inhaltlichen Dimension einer Vorlesung über „Didaktik“ ein Feedback geben, wenn er nicht über Grundzüge des Verständnisses von Didaktik verfügt. Oder wie kann ein Politiker Sachfragen beantworten, wenn er keine fachliche Kompetenz besitzt. In diesem Fall greift er, in der Regel, auf seine Soft Skills zurück und plaudert über etwas, worüber er als Person individuell verfügt. Dies ist von Person zu Person, von Fall zu Fall, unterschiedlich. So werden Fragen mit Fragen beantwortet oder es wird eine Aussage zu einem anderen Thema gemacht, für das man fachlich befähigt ist, dass aber mit dem situativen Thema gar nichts zu tun hat. Es zeigt sich hier z.B. die personengebundene Befähigung (Soft Skills) zur Kommunikativen Kompetenz lediglich auf der Beziehungsebene. Menschen, die von der Sache her nicht überzeugen können, flüchten geradezu in die Abstraktheit von Soft Skills, um im Falle einer Kritik über ihre fachliche Nichtbildung, sich an dem bedeutsamen Ausmaß ihrer eigenen „weichen“ Bildungsfaktoren messen und bewerten lassen zu wollen. Die Bewertung von Soft Skills und fachlicher Bildung ist ein Problem von Macht oder Ohnmacht derjenigen, die definieren und bewerten. Dieser Prozess geht bis in die Disziplinen der einzelnen Wissenschaften.

Soft Skills sind oft das Ergebnis fachwissenschaftlicher Vorstellungen, Ideen und Theorien und deren anthropologischer Entwürfe. Sie stellen sowohl personale Kompetenzen als auch auf Personen bezogene Qualifikationsstandards dar.

Ohne eine neue Diskussion über die Bedeutung der Begrifflichkeit von Kompetenzen und Qualifikationen zu führen kann man davon ausgehen, „dass im Allgemeinen sowohl Qualifikations- als auch Kompetenzansätze im betrieblichen Alltag eine bedeutende Rolle spielen werden. Um Anforderungsprofile in Stellenanzeigen zu entwerfen, stellen der Kompetenzbegriff ebenso wie der Qualifikationsbegriff akzeptierte sprachliche Ausdrucksformen zur Verfügung." (Sailer, S. 36) 


\section{Thesen zur Dialektik von Soft Skills und Fachlicher Bildung Thesen:}

1. Soft Skills sind normale Bildungskategorien und Teil einer Allgemeinen Bildung. Das Verständnis, was unter Allgemeiner Bildung zu verstehen ist, ist zeitabhängig. Darauf verweist auch die Diskussion über Soft Skills.

2. Soft Skills sind als personalgebundene Kriterien auch immer schon Gegenstand bildungstheoretischer Debatten.

3. Ausrichtung auf Soft Skills

- ist die Folge des Verlustes von fachlich orientierten Ansprüchen;

- ist die Folge der Suche nach dem Allgemeinen von Bildung

(Allgemeiner Bildung). Z.B. wird bei den Lehrämtern das eigentliche Fach (z.B. Germanistik) vernachlässigt, zugunsten vermittlungsmethodischer bzw. lernmethodischer Ansätze (Fachdidaktik Deutsch). Hier zeigt sich eine Tendenz, die sich von der fachlichen Bildung zu den Soft Skills (Methodenkompetenz) hin orientiert;

- ist die Folge eines Versuches, über Soft Skills die geringer werdenden Halbwertzeiten fachlicher Bildungskategorien zu korrigieren, wobei die sachlich orientierte Kompetenz als fachliche Bildungskategorie gelten kann

- Diesem Ansatz liegt eine falsche Annahme zu Grunde.

Denn auch die Soft Skills unterliegen den abnehmenden Halbwertzeiten. Dies sogar aus zweierlei Gründen: Erstens durch ihre Koppelung an die Kategorien fachlicher Kompetenz, wodurch sie sich inhaltlich begreifen lassen, und zweitens durch die Veränderung anthropologischer und kultureller Vorstellungen und Trends.

4. Soft Skills ist eine trendorientierte Bezeichnung für Bildung. Immer dann, wenn in der Diskussion das Wort „Bildung“ möglichst vermieden werden soll, wählt man andere Bezeichnungen wie z.B. Charaktereigenschaften, Tugenden Sittlichkeit oder sogar EQ (emotionale Intelligenz). Dabei ist man auf der Suche nach einem Multiplikator, der auf Wissen, materiale und sachbezogene Fähigkeiten und Fertigkeiten steuernd oder sogar gewinnbringend (erfolgreich) in Lebenssituationen wirkt und darüber hinaus ein entscheidender Indikator sein könnte für Erfolg oder Misserfolg, fürs Gelingen oder Misslingen von Handlungsabläufen. 
5. Soft Skills sind Ausdruck von Vorstellungen, Ideen und Theorien (Schulkultur, Unternehmenskultur, Unternehmensphilosophie)

6. Soft Skills erklären sich aus Notwendigkeiten des täglichen Lebens und sind von daher einem Wandel unterzogen (u.a. Halbwertzeiten).

7. Soft Skills sind individuelle, personengebundene Befähigungen. Sie erklären sich über bestimmte Inhalte, Informationen und Wissensstrukturen. Soft Skills sind selbständige Bildungskategorien.

8. Soft Skills sind formale Bildungskategorien.

9. Soft Skills sind spezielle, personengebundene Bildungskategorien Soft Skills sind fachlich Übergreifend

10. Soft Skills werden konkret durch fachliche Bildungskategorien (Zur Dialektik von Soft Skills und fachlicher Bildung).

11. Soft Skills sind als Einstellungen, Haltungen und Gesinnungen nicht allgemein zu Verstehen, sondern sie sind typisch, von der spezifischen Aktivität, dem Tun abhängig und über die fachliche Kompetenz erklär- und verstehbar.

12. Soft Skills als spezielle Bildungskategorien und/oder fachliche Bildung als spezielle Bildungskategorien, sind in der Synthese Allgemeine Bildungskategorien. Der Allgemein Gebildete Mensch ist in der Lage, auf neue (spezielle Soft Skills) und veränderte Anforderungen (spezielle fachliche Kategorien) selbständig und autonom Einfluss nehmen zu können.

\section{Literatur:}

Malik, F.: Führen, Leisten, Leben; München 2001

Sailer, M.: Anforderungsprofile und akademischer Arbeitsmarkt; Münster 2009

Ryschka, J., Solga, M., Mattenklott, A.: Praxishandbuch Personalentwicklung. Instrumente, Konzepte, Beispiele; Wiesbaden 2008; 2. Auflage Timmermann, D.: Bildungsökonomie; in: Tippelt, R. (Hrsg.) Handbuch Bildungsforschung (S. 81 - 122) Opladen 2002 